Niamien-Ebrottie et al J. Appl. Biosci. 2013. Composition et variation spatio-saisonnière du

\title{
Composition et variation spatio-saisonnière du peuplement algal des rivières côtières du Sud-est de la Côte d'Ivoire
}

\author{
Niamien-Ebrottié Julie Estelle ${ }^{1}$, Konan Koffi Félix ${ }^{2-*}$, Edia Oi Edia1 ${ }^{1}$ Ouattara Allassane ${ }^{1}$, Gourène \\ Germain ${ }^{1}$ \\ ${ }^{1}$ Laboratoire d'Environnement et de Biologie Aquatique, UFR - Sciences et Gestion de l'Environnement, Université \\ Nangui Abrogoua, Abidjan, Côte d'Ivoire \\ 2 UFR - Environnement, Université Jean Lorougnon Guédé, Daloa, Côte d'Ivoire \\ * Auteur correspondant: Konan Koffi Félix; BP 150 Daloa, Côte d'Ivoire ; GSM : 0022506216160 ; E-mail : \\ konanfelix@yahoo.fr
}

Original submitted in on 10 th June 2013 Published online at www.m.elewa.org on 30 th June 2013.

https://dx.doi.org/10.4314/iab.v66i0.95012

\section{RESUME}

Objectif : La composition et la distribution spatio-temporelle des micro-algues de quatre rivières du Sud-est de la Côte d'Ivoire (Soumié, Eholié, Ehania et Noé) ont été analysées, en relation avec des paramètres physicochimiques du milieu.

Méthodologie et résultats : La zone pélagique et les supports littoraux (pierres, feuilles et bois) ont été échantillonnés en amont et en aval de chaque rivière. Au total, 192 taxons spécifiques et infraspécifiques ont pu être identifiés, parmi lesquels environ $75 \%$ d'espèces de Diatomées, $10,4 \%$ de Cyanobactéries, 9,9\% de Chlorophytes, 4,2 \% d'Euglénophycées et 0,5\% de Rhodophycées. Les Diatomées constituent la classe la plus constamment rencontrées dans les prélèvements des quatre cours d'eau De plus les algues périphytiques sont plus diversifiées que celles de pleine eau. Les espèces Gyrosigma acuminatum, Nitzschia palea, Ulnaria biceps et Gomphonema parvulum sont communes à tous les habitats explorés. Conclusion : Les variations de la composition des peuplements entre les rivières sont relativement faibles, avec des diversités plus importantes dans la rivière Noé (134 taxons) et peu élevées dans la rivière Ehania (127 taxons). En outre, les algues récoltées sur feuille et sur bois présentent une forte similarité dans tous les cours d'eau prospectées. Mots clés : Phytoplancton, périphyton, diversité taxonomique, Rivières côtières, Côte d'Ivoire.

\section{ABSTRACT \\ Composition and spatio-seasonal variation of algal assemblage of coastal rivers in Southeastern of Ivory Coast. \\ Objective: The composition and the spatio-seasonal distribution of micro-algae in combination with water physico-chemical variables were measured in four coastal rivers of the Southeast of Ivory Coast (Soumié, Eholié, Ehania and Noé) from upstream to downstream.}


Methodology and results: Samplings were carried out seasonally, at two sampling sites (upstream, downstream) and at pelagic area and inshore of each river (stones, leaves and pieces of branches). One hundred and ninety-three (192) species and infra-species were identified. This micro-algae population contained $75 \%$ of Diatoms, $10.4 \%$ of Cyanobacteria, $9.9 \%$ of Chlorophyta, $4.2 \%$ of Euglenophyceae and 0.5 $\%$ of Rhodophyceae. Diatoms are also the class most constantly found in the samples from these four coastal rivers. Periphytic algae are more diversified than those of open water are. Gyrosigma acuminatum, Nitzschia palea, Ulnaria biceps and Gomphonema parvulum species are common to all habitats explored. Conclusion: The composition of algae showed slight differences between rivers. The highest diversity was observed at Noé River (134 taxa) and the lowest at Ehania River (127 taxa).

Key words: Phytoplankton, periphytic, taxonomic diversity, coastal rivers, Ivory Coast.

\section{INTRODUCTION}

Les zones côtières au Sud-est de la Côte d'lvoire sont des lieux de concentrations humaines où les impératifs liés au développement de l'agriculture menacent l'équilibre des petits écosystèmes fluviaux côtiers. Cette région caractérisée par un substrat géologique de type ferralitique typique remanié est influencée par un climat équatorial humide caractérisé par deux saisons de pluies et deux saisons sèches : les saisons de pluies s'étendent d'avril à juillet pour la grande saison et d'octobre à novembre pour la petite ; les petite et grande saisons sèches couvrent les périodes allant respectivement d'août à septembre et de décembre à mars (Eldin, 1971). La crue la plus importante s'observe en juinjuillet, suivie d'une seconde de moindre intensité en octobre-novembre (Girard et al., 1971 ; Durand et Guiral, 1994). Bien arrosée avec une pluviométrie annuelle variant entre 1000 et $1500 \mathrm{~mm}$ (Anonyme $1,2005)$, cette zone est drainée par de nombreux cours d'eau côtiers. En effet, cette région est connue depuis plusieurs décennies pour sa forte productivité agricole (plantations industrielles et villageoises de caféiers, cacaoyers, ananas, palmiers à huile). Ces plantations occupent environ $44 \%$ de la superficie de cette région (Anonyme 2, 2001). Or, la production de ces cultures implique une utilisation de produits phytosanitaires (engrais, pesticides), ce qui pourrait avoir des conséquences sur la qualité des eaux, et conduire à des changements profonds de la composition et de la structure des peuplements des organismes des hydrosystèmes de cette zone. La prise en compte des altérations provoquées par les activités humaines sur ces cours d'eau apparaît actuellement comme une préoccupation majeure. $\mathrm{Ce}$ contexte impose aujourd'hui une approche pluridisciplinaire des effets anthropiques associant étude des composantes biologiques et physicochimiques. La communauté algale représente, du point de vue abondance et biomasse, une composante essentielle des milieux aquatiques. Elle constitue, par sa concentration sur les substrats immergés ou en suspension dans la colonne d'eau, une source nutritionnelle pour de nombreux organismes aquatiques tels que le zooplancton, les insectes et certains poissons, notamment les filtreurs et les brouteurs (Large et al., 1993 ; Round, 1993). En outre, de nombreux travaux ont montré que dans des conditions de stabilité et d'enrichissement en substances nutritives favorables, une population d'algues peut se développer et, selon les cas, être bénéfique ou préjudiciable pour l'Homme (Carmichael, 1994 ; Kotak et al., 1996). En outre, plusieurs indices, basés sur la sensibilité ou la tolérance à la pollution de certaines espèces d'algues microscopiques (Diatomées, notamment), ont été mis au point et utilisés en routine pour la surveillance biologique des réseaux hydrographiques (Prygiel et al., 1996 ; Kelly, 1997 ; Prygiel \& Coste, 2000). Malgré leur importance dans les hydrosystèmes, les algues restent en général très peu étudiées en Afrique, surtout dans les écosystèmes d'eau douce où les travaux les plus marquants sont ceux effectués par Compère pendant les années 1970 (cf. Compère, 1975; Compère, 1977). En Côte d'Ivoire, les travaux réalisés concernent des aspects systématiques ( $\mathrm{Da}$ et al., 1999 ; Da et al., 2009 ; Ouattara et al., 2000) et écologiques (Iltis, 1982 ; Ouattara et al., 2003 ; 
Ouattara et al, 2007) des peuplements algaux. Cependant, en dehors des travaux de taxonomie de Ouattara et al. (2000) et Da (2007) sur les algues planctoniques des cours d'eau, une seule étude lltis (1982) a été effectuée sur les algues périphytiques des eaux courantes. Ainsi, la composition de cette communauté algale reste peu connue dans les cours d'eau de Côte d'Ivoire et particulièrement dans les zones à forte activité agricole comme la région du
Sud-est. De ce fait, la taxonomie et l'écologie des algues de cette région demandent à être caractérisées. Le présent travail a pour objectif général d'étudier les algues périphytiques et phytoplanctoniques de quatre rivières du Sud-est de la Côte d'Ivoire. Plus spécifiquement, il s'agit d'identifier et analyser la composition des algues (périphyton, phytoplancton).

N1 et N2) (Figure 1). Ces stations ont été choisies en tenant compte de la zonation hydrologique et de l'accessibilité.

\section{MATERIEL ET METHODES}

Description des stations d'échantillonnage: Deux stations de prélèvement ont été retenues sur chacune des rivières Soumié (stations $\mathrm{S} 1$ et $\mathrm{S} 2$ ), Eholié (stations E1 et E2), Ehania (stations Eh1 et Eh2) et Noé (stations

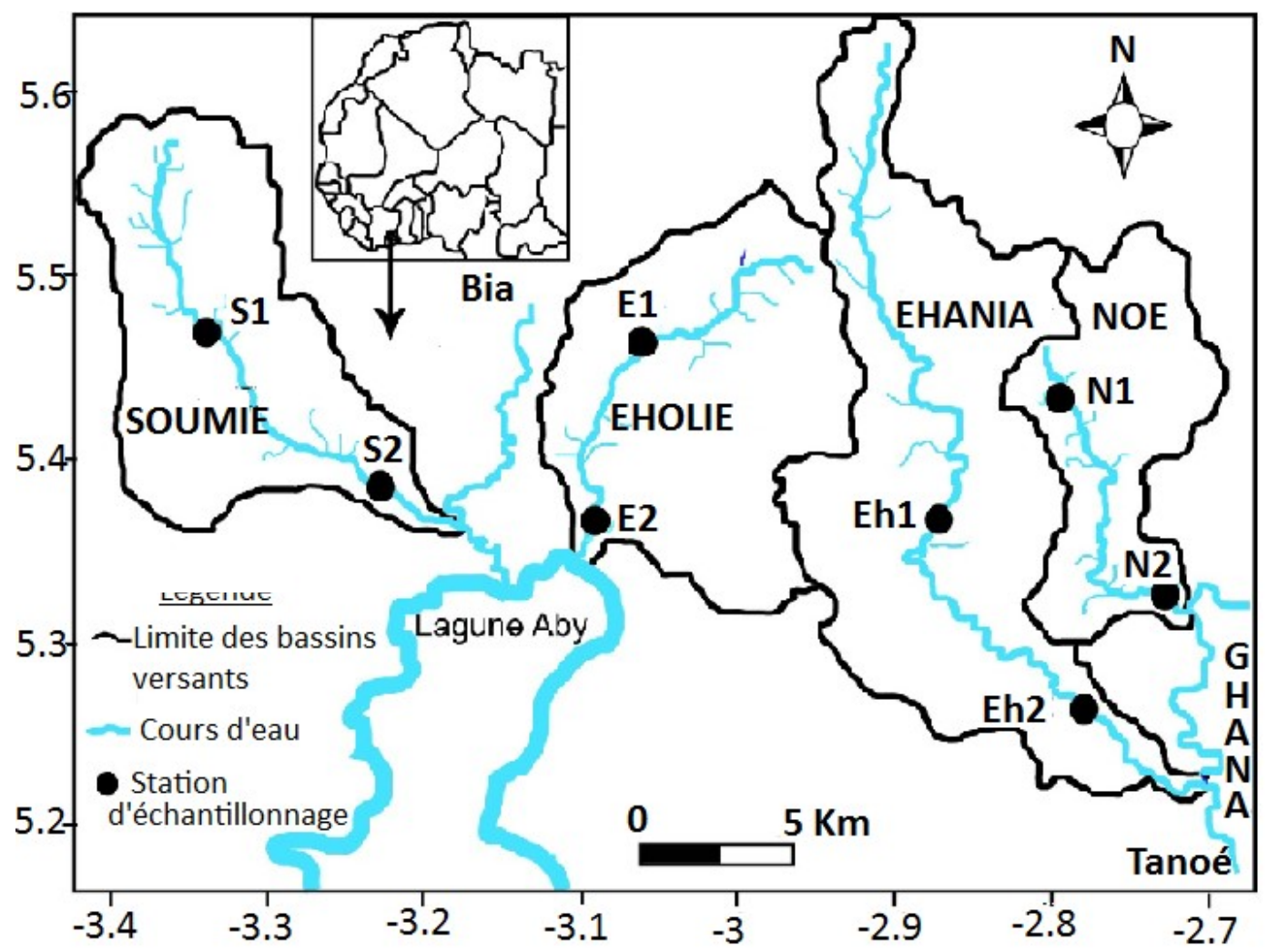

Figure1: Localisation des rivières et des stations d'échantillonnage (modifiée de Konan et al., 2006) ; $S=$ Soumié, $E=$ Eholié, Eh = Ehania, $\mathrm{N}=$ Noé, 1 = cours supérieur, 2 = cours inférieur.

Mesures des paramètres abiotiques : un navigateur GPS MLR SP12X a servi à la localisation des stations. La conductivité a été mesurée à l'aide d'un conductimètre de type hach, modèle 44600. Un pH-mètre de type WTW a permis de mesurer le ph et la température de l'eau. La mesure de l'oxygène dissous a été réalisée au moyen d'un oxymètre de type WTW. Un disque de Secchi a servi à la détermination de la transparence. Une sonde master a permis de jauger la profondeur des cours d'eau. Cinq flotteurs, un chronomètre et un décamètre ont été utilisés pour la détermination de la vitesse du courant. Le dosage des nitrates a été effectué à l'aide d'un spectrophotomètre de marque Shimadzu uv1205. Huit campagnes d'échantillonnage ont été réalisées de juillet 
2003 à mars 2005. La conductivité (CND), le ph, la température, l'oxygène dissous (OD), la transparence, la profondeur et la vitesse du courant ont été mesurés in situ entre 08 heures et 10 heures du matin aux différentes stations. Pour la concentration en nitrates, un prélèvement d'eau de surface a été effectué et conservé dans une bouteille de 1 litre à une température de $4^{\circ} \mathrm{C}$. $\mathrm{Au}$ laboratoire, la concentration en nitrates a été déterminée selon la norme t90-110 (afnor, 1994). La canopée et la composition du substrat ont été évaluées visuellement et exprimées en pourcentage de couverture du site selon les méthodes de Arab et al. (2004).

Prélèvement et d'observation des microalgues. : Les algues pélagiques ont été prélevées à l'aide d'une bouteille hydrologique de type Van Dorn de capacité 2,5 litres et d'un filet à plancton. Concernant le filet à plancton, une traction sur une distance de $10 \mathrm{~m}$ a été effectuée avec le filet. Le périphyton a été recueilli au moyen d'une brosse à poils bien séparés sur divers supports immergés organiques (feuilles, troncs d'arbres morts) et inorganiques (graviers et galets). Les substrats inorganiques ont été échantillonnés seulement sur les rivières Ehania et Noé. Une surface de $10 \mathrm{~cm}^{2}$ a été lavée avec la brosse. Les échantillons ont été conservés dans un pilulier de $30 \mathrm{~mL}$ et fixés au formol $5 \%$. De l'acide nitrique technique, une plaque chauffante et une centrifugeuse ont été utilisés pour le nettoyage des frustules des Diatomées, selon la méthode de Prygiel et al. (2000). L'observation des taxons a été effectuée à l'aide d'un microscope trinoculaire de type Olympus BX40 muni d'une chambre claire après montages entre lames et lamelles. L'identification des taxons a été effectuée jusqu'au niveau spécifique ou infraspécifique à l'aide des travaux (clés et/ou description) de plusieurs auteurs dont Desikachary (1959), Foged (1966), Prescott et al. (1975), Compère $(1975 ; 1977 ; 1980 ; 1991 ; 2000)$, Krammer \& Lange-bertalot (1986), Carter \& Denny (1987), Cocquyt (1998), Komárek \& Anagnostidis (1999 et 2005), Sophia

\section{RESULTATS}

Environnement physico-chimique des eaux: Les valeurs de $\mathrm{pH}$ oscillant autour de la neutralité présentent de très faibles variations de l'amont à l'aval et d'une saison à l'autre dans les rivières Soumié, Eholié, Noé et Ehania (Tableau I). Dans toutes les rivières, les concentrations en oxygène dissous en aval sont sensiblement supérieures aux valeurs enregistrées en amont. Concernant le débit, il présente une faible variation d'une station à l'autre et d'une saison à l'autre et al. (2005), Ouattara et al. (2000), Prygiel \& Coste (2000), Guiry \& Guiry (2007) et Jahn \& Kusber (2007). Caractérisation du peuplement algal : Le pourcentage d'occurrence qui renseigne sur les préférences d'habitat d'une espèce donnée a été déterminé selon Hyslop (1980) et Dajoz (2000) comme suit :

$F=\frac{F_{i} \times 100}{F_{t}}$

$F_{i}=$ nombre de relevés contenant l'espèce $i ; F_{t}=$ nombre total de relevés effectués. En fonction de la valeur de $F$, trois groupes d'espèces sont distingués: espèces constantes $(F \geq 50 \%)$; espèces accessoires ( $25 \% \leq F<$ $50 \%)$; espèces accidentelles $(F<25 \%)$.

La similarité déterminant la ressemblance entre deux communautés (habitats) a été estimée par l'indice de similarité de Sorenson :

$$
C s=\frac{2 j}{a+b_{t}}
$$

$a=$ nombre d'espèces présentes dans le milieu $A ; b=$ nombre d'espèces présentes dans le milieu $B ; j=$ nombre d'espèces communes aux 2 milieux. L'indice de similarité de Sorenson varie de 0 (absence de similarité) à 1 (milieux identiques).

Traitements statistiques: Une analyse en Composantes Principales (ACP) a été utilisée pour ordonner les stations en fonction des paramètres abiotiques. L'Analyse Factorielle des Correspondances $(\mathrm{AFC})$ a été appliquée à la composition du peuplement afin de regrouper les stations d'échantillonnage en fonction de leurs similarités floristiques. Ces analyses ont été effectuées avec le logiciel $R$ version 2.0.1 (lhaka \& Gentleman, 1996) muni du package ADE 4 (Thioulouse et al., 1997). Le test de Kruskal-Wallis a permis de tester la variabilité spatio-temporelle des indices entre les différents groupes de stations. Le test de Mann-Whitney a ensuite été utilisé pour identifier les variations entre les groupes pris deux à deux. Le seuil de significativité de ces deux derniers tests est de $5 \%$.

dans toutes les rivières. Les débits les plus élevés sont enregistrés pendant la petite saison de pluies dans toutes les rivières et les plus faibles débits pendant la grande saison de pluies et la grande saison sèche. Les concentrations en nitrate dans toutes les rivières sont plus faibles en amont par rapport aux stations en aval. Les concentrations les plus élevées de nitrates sont enregistrées pendant la grande saison de pluies à toutes les stations des rivières étudiées excepté la station en 
Niamien-Ebrottie et al J. Appl. Biosci. 2013. Composition et variation spatio-saisonnière du peuplement algal des rivières côtières la Cote d'Ivoire

aval de Noé où elles sont observées en grande saison sèche. Quant aux valeurs de conductivité obtenues, elles sont plus élevées en amont qu'en aval de toutes les rivières prospectées excepté la rivière Eholié où la variation est plus faible. La variation saisonnière est très peu marquée pour ce paramètre. 
Tableau 1 : Valeurs des paramètres physico-chimiques (oxygène dissous, transparence, débit, concentration en nitrate, conductivité et pH) de l'eau des rivières.

\begin{tabular}{|c|c|c|c|c|c|c|c|c|c|c|}
\hline Rivière & Stations & Saisons & $\begin{array}{c}\text { Température } \\
\left({ }^{\circ} \mathrm{C}\right)\end{array}$ & $\mathrm{pH}$ & $\begin{array}{c}\text { Oxygène } \\
\text { dissous (mg/L) }\end{array}$ & $\begin{array}{c}\text { Transparen } \\
\text { ce (m) }\end{array}$ & $\begin{array}{l}\text { Débit } \\
\left(\mathrm{m}^{3} / \mathrm{s}\right)\end{array}$ & $\begin{array}{l}\text { Nitrate } \\
\text { (mg/L) }\end{array}$ & $\begin{array}{c}\text { Conductivité } \\
(\mu \mathrm{S} / \mathrm{cm})\end{array}$ & Profondeur (m) \\
\hline \multirow{8}{*}{ Soumié } & \multirow{4}{*}{ S1 } & GSP & 24,40 & 7,48 & 4,46 & 0,61 & 0,33 & 1,93 & 60,80 & 1,23 \\
\hline & & PSS & 24,47 & 7,13 & 4,17 & 0,58 & 0,34 & 1,64 & 58,47 & 0,66 \\
\hline & & PSP & 26,40 & 6,87 & 3,55 & 0,82 & 0,38 & 1,49 & 55,90 & 0,53 \\
\hline & & GSS & 25,75 & 6,76 & 5,27 & 0,54 & 0,28 & 1,33 & 53,20 & 0,89 \\
\hline & \multirow{4}{*}{ S2 } & GSP & 25,30 & 6,77 & 5,99 & 0,64 & 0,21 & 3,42 & 45,35 & 1,49 \\
\hline & & PSS & 24,43 & 7,04 & 4,50 & 0,75 & 0,33 & 2,39 & 42,23 & 1,22 \\
\hline & & PSP & 27,40 & 6,94 & 5,05 & 0,48 & 0,34 & 2,27 & 45,00 & 1,45 \\
\hline & & GSS & 25,60 & 6,58 & 5,83 & 0,39 & 0,26 & 2,14 & 39,10 & 1,59 \\
\hline \multirow{8}{*}{ Eholié } & \multirow{4}{*}{ E1 } & GSP & 25,00 & 6,87 & 6,08 & 0,44 & 0,30 & 1,91 & 52,30 & 1,71 \\
\hline & & PSS & 25,40 & 7,06 & 5,98 & 0,55 & 0,30 & 1,57 & 57,40 & 1,09 \\
\hline & & PSP & 26,30 & 6,88 & 5,04 & 0,47 & 0,38 & 1,62 & 55,50 & 0,70 \\
\hline & & GSS & 26,75 & 7,02 & 6,42 & 0,60 & 0,30 & 1,88 & 24,55 & 1,38 \\
\hline & \multirow{4}{*}{ E2 } & GSP & 25,05 & 7,11 & 8,43 & 0,56 & 0,40 & 2,97 & 59,95 & 1,65 \\
\hline & & PSS & 25,37 & 6,89 & 6,79 & 0,55 & 0,53 & 2,74 & 57,23 & 2,09 \\
\hline & & PSP & 26,60 & 6,94 & 8,71 & 0,47 & 0,63 & 2,62 & 55,90 & 2,1 \\
\hline & & GSS & 27,10 & 6,94 & 5,46 & 0,49 & 0,41 & 2,61 & 54,15 & 1,74 \\
\hline \multirow{8}{*}{ Ehania } & \multirow{4}{*}{ Eh1 } & GSP & 24,60 & 7,09 & 5,28 & 0,79 & 0,14 & 1,57 & 67,80 & 1,25 \\
\hline & & PSS & 25,27 & 6,92 & 6,09 & 0,76 & 0,44 & 1,03 & 64,17 & 1,29 \\
\hline & & PSP & 26,20 & 7,16 & 4,92 & 0,62 & 0,17 & 1,51 & 60,40 & 0,80 \\
\hline & & GSS & 26,10 & 7,15 & 4,05 & 0,72 & 0,22 & 1,59 & 62,15 & 0,97 \\
\hline & \multirow{4}{*}{ Eh2 } & GSP & 25,15 & 6,74 & 7,98 & 0,48 & 0,25 & 2,16 & 51,75 & 2,56 \\
\hline & & PSS & 25,20 & 7,05 & 6,78 & 0,50 & 0,34 & 1,46 & 56,27 & 2,25 \\
\hline & & PSP & 26,70 & 6,74 & 9,82 & 0,45 & 0,40 & 1,95 & 49,60 & 2,25 \\
\hline & & GSS & 26,95 & 6,90 & 6,52 & 0,53 & 0,18 & 2,16 & 58,50 & 2,11 \\
\hline \multirow{8}{*}{ Noé } & \multirow{4}{*}{ N1 } & GSP & 24,95 & 7,13 & 6,29 & 0,60 & 0,29 & 1,60 & 68,00 & 0,78 \\
\hline & & PSS & 25,03 & 7,00 & 7,63 & 0,59 & 0,53 & 1,39 & 63,73 & 0,64 \\
\hline & & PSP & 26,10 & 6,58 & 7,85 & 0,52 & 0,28 & 1,36 & 64,80 & 0,70 \\
\hline & & GSS & 25,95 & 7,12 & 7,17 & 0,60 & 0,30 & 1,61 & 60,90 & 0,67 \\
\hline & \multirow{4}{*}{ N2 } & GSP & 25,10 & 6,92 & 7,32 & 0,44 & 0,49 & 2,11 & 54,60 & 2,25 \\
\hline & & PSS & 25,50 & 6,67 & 6,80 & 0,46 & 0,54 & 2,10 & 54,47 & 2,13 \\
\hline & & PSP & 27,60 & 5,76 & 9,82 & 0,37 & 0,30 & 2,18 & 53,30 & 3,86 \\
\hline & & GSS & 26,40 & 6,83 & 6,67 & 0,44 & 0,23 & 3,15 & 52,95 & 2,15 \\
\hline
\end{tabular}


Niamien-Ebrottie et al J. Appl. Biosci. 2013. Composition et variation spatio-saisonnière du peuplement algal des rivières côtières la Cote d'Ivoire

$S=$ Soumié $; E$ = Eholié $; E h=$ Ehania $; N=$ Noé $; 1=$ amont $; 2$ = aval $; G S P=$ grande saison des pluies (avril-juillet) $; P S S=$ petite saison sèche (août-septembre) ; $\mathrm{PSP}=$ petite saison des pluies (octobre-novembre) $; \mathrm{GSS}=$ grande saison sèche (décembre-mars). 
Typologie abiotique des stations : Les deux premiers axes de l'Analyse en Composantes Principales (ACP) exprimant $55,5 \%$ de l'inertie totale du tableau de données (Figure $2 \mathrm{~A}$ ) ont été retenus pour l'expression des résultats de l'ACP. Le cercle de corrélation (Figure 2B) montre que l'axe 1 est corrélé négativement à la conductivité, au pH et à la transparence. La teneur en nitrates, la profondeur, l'oxygène dissous et le pourcentage de galets sont positivement corrélés à l'axe
1. L'axe 2 est expliqué positivement par le substrat graveleux et négativement par le pourcentage de vase et la canopée. La carte factorielle indique que l'axe 1 met en évidence un gradient amont-aval de minéralisation (Figure 2C). Les premières stations en amont présentent des eaux relativement plus minéralisées (conductivité, transparence et pH plus élevés). Les stations en aval présentant des profondeurs plus importantes, ont des concentrations en nitrate plus élevées.
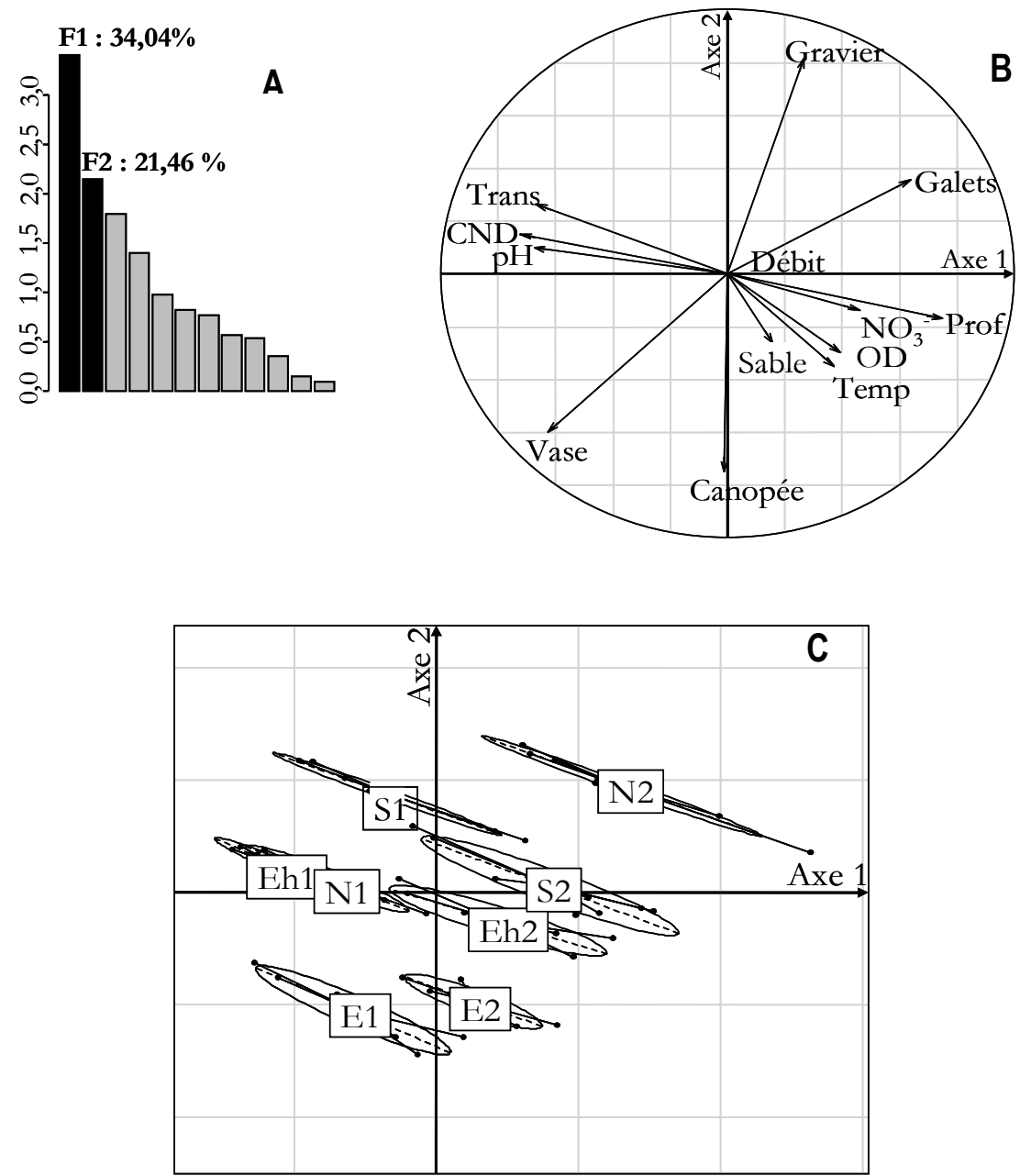

Figure 2 : Analyse en Composantes Principales réalisée à partir des paramètres physiques, chimiques et mésologiques des stations ; $A=$ Valeurs propres des axes factoriels $; B=$ Cercles de corrélation, $C=$ Cartes factorielles des stations sur les plans factoriels F1 $\mathrm{F} 2 ; \mathrm{S}=$ Soumié $; \mathrm{E}=$ Eholié $; \mathrm{Eh}=$ Ehania $; \mathrm{N}=$ Noé $; 1=$ amont $; 2=$ aval $;$ Temp = température, $\mathrm{NO}_{3}{ }^{-}=$nitrate, $\mathrm{CND}=$ conductivité $;$ Trans $=$ transparence $; \mathrm{OD}=0$ oxygène dissous ; Prof $=$ profondeur.

Analyse du peuplement algal : Un total de 192 taxons (espèces et variétés) répartis en 68 genres, 9 ordres, 6 classes et 5 embranchements a été inventorié dans l'ensemble des quatre rivières. Les embranchements rencontrés sont: Hétérokontophytes,
Cyanobactéries, Chlorophytes, Euglénophytes et Rhodophytes. Les Hétérokontophytes, avec la classe des Diatomées, sont les plus diversifiées avec 144 taxons (75 $\%$ ). Dans cette classe, les formes pennées, sont les mieux représentées avec $94,5 \%$ des taxons. Après les 
Hétérokontophytes viennent les Cyanobactéries et les Chlorophytes avec respectivement 20 et 19 taxons, soit $10,4 \%$ et $9,9 \%$ (Figure 3). Ces trois embranchements renferment $94,1 \%$ des genres et $95,3 \%$ des taxons (espèces et variétés) inventoriés. Par ailleurs, l'inventaire des microalgues des quatre rivières indique que le nombre de taxons par rivière est compris entre 127 (rivière Éhania) et 134 (rivière Noé). Les stations en amont des rivières Soumié, Ehania et Noé, ainsi que la station aval de la rivière Éholié sont les plus diversifiées avec respectivement 106, 109, 104 et 107 taxons. Avec 88 taxons recensés, la station Eh2 en aval de la rivière Ehania est la plus pauvre en termes de diversité.

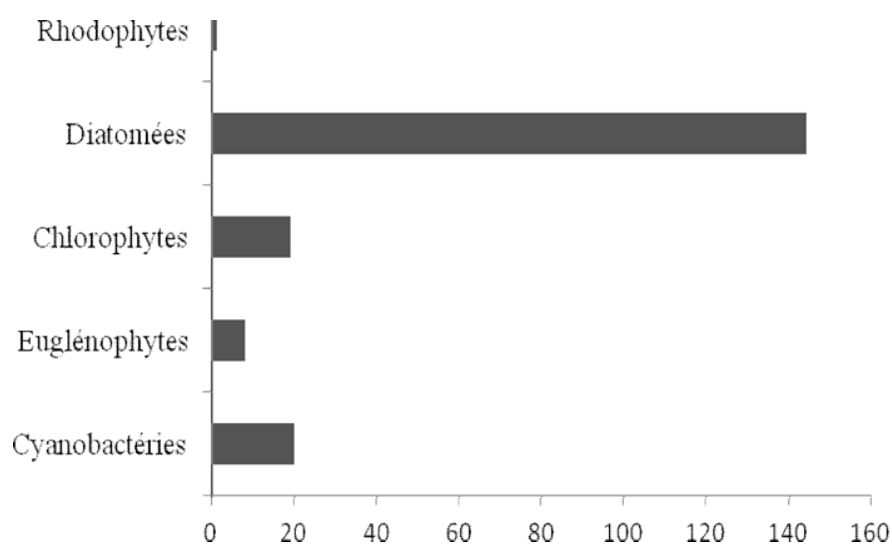

Figure 3 : Proportion des différents groupes d'algues dans les rivières

Dans toutes les rivières le nombre de taxons constants est très faible par rapport aux taxons accessoires et accidentels. Dans la rivière Soumié, les espèces constantes sont Planothidium lanceolatum, Eunotia incisa et Ulnaria biceps en amont et $E$. incisa en aval. La rivière Eholié présente 2 espèces constantes : Nitzschia palea en amont et Navicula menisculus en aval. Pour ce qui est de la rivière Ehania, 2 espèces ( $N$. palea et Gyrosigma acuminatum) sont constantes à la station amont (Eh1) contre un seul taxon constant (Planothidium rostratum) à la station en aval (Eh2). Concernant la rivière Noé, deux (U. biceps et $N$. palea) et une (N. palea) espèces constantes ont été rencontrées respectivement en amont et en aval. La répartition des taxons en fonction de ces habitats indique que le nombre de taxons inventoriés pour le périphyton (feuilles, bois, pierres) est largement supérieur à celui du phytoplancton quelle que soit la rivière considérée. En outre, parmi les espèces inventoriées, quatre (Gomphonema parvulum, Gyrosigma acuminatum, Nitzschia palea, Ulnaria biceps) sont communes à tous les habitats dans l'ensemble des rivières étudiées. Les similarités de la composition taxonomique entre les types d'habitats dans les rivières étudiées ont été déterminées (Tableau 2 ; Figure 4). Dans la rivière Soumié, 10 taxons ont été rencontrés exclusivement sur feuilles, 37 sur bois et 24 en pleine eau. Ces trois types d'habitats ont en commun 21 taxons.
Les habitats du périphyton (feuilles et bois) présentent le maximum de taxons en commun (14) avec une valeur de 0,50 de l'indice de similarité, par contre les communautés de pleine eau et celles colonisant les feuilles enregistrent le minimum de taxons en commun (4 taxons). Pour ce qui concerne la rivière Eholié, les taxons spécifiques à chaque habitat sont au nombre de 26 sur feuilles et également dans la pleine eau et de 10 sur bois. Ces trois habitats ont en commun 24 taxons. Par ailleurs, le nombre le plus élevé de taxons en commun (42) est observé entre les échantillons récoltés sur feuilles et sur bois $(C s=0,74)$ et le plus faible effectif $(4)$ de taxons en commun a été relevé entre les communautés de pleine eau et celles fixées sur bois (Cs $=0,37)$. Au niveau de la rivière Ehania, 12, 4, 33 et 4 taxons spécifiques ont été inventoriés respectivement en pleine eau, sur bois, sur feuilles et sur pierres. Tous ces habitats ont en commun 17 taxons. En outre, le plus grand nombre de taxons en commun est rencontré entre les communautés d'algues fixées sur feuilles et sur bois ( 13 taxons; $C s=0,56$ ) et le plus petit nombre entre les taxons récoltés sur bois et sur pierres ( 1 taxon ; $C s=0,46)$. Concernant la rivière Noé, elle est caractérisée par 34, 6, 23 et 6 taxons recensés exclusivement dans la pleine eau, sur bois, sur feuilles et sur pierres. Ces habitats ont en commun 12 taxons. Le minimum de taxons en commun est 2 et le maximum est 19 (taxons recensés sur feuilles et bois ; $\mathrm{Cs}=0,58$ ). 

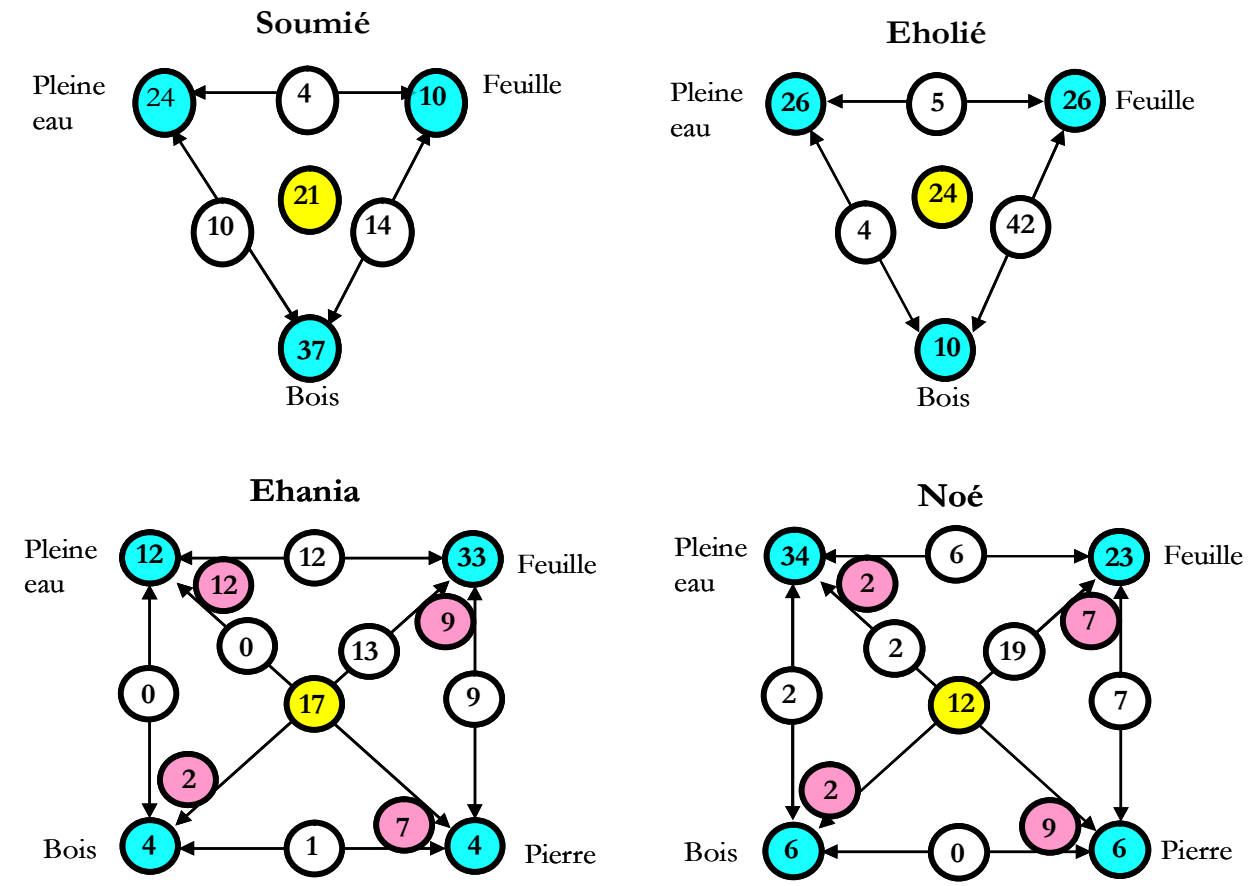

8

Nombre de taxons spécifiques à un habitat Nombre de taxons communs à trois habitats

Nombre de taxons communs à deux habitats Nombre de taxons communs à tous les habitats

Figure 4 : Similarités de la composition taxonomique entre les différents types d'habitats des rivières Soumié, Eholié, Ehania et Noé (le nombre de taxons communs à trois habitats est indiqué à l'angle droit de chaque triangle rectangle que forme les trois habitats concernés).

Tableau 2 : Valeurs de l'indice de similarité de Sorensen des habitats dans les différentes rivières

\begin{tabular}{clc}
\hline Rivières & \multicolumn{1}{c}{ Habitats } & Indice de Sorensen (Cs) \\
\hline \multirow{3}{*}{ Soumié } & 0,44 \\
& Feuilles-Pleine eau & 0,50 \\
& Feuilles- Bois & 0,42 \\
\hline \multirow{3}{*}{ Éholié } & Pleine eau -Bois & 0,35 \\
& Feuilles-Pleine eau & 0,74 \\
& Feuilles- Bois & 0,37 \\
\hline & Pleine eau -Bois & 0,54 \\
\multirow{2}{*}{ Éhania } & Feuilles-Pleine eau & 0,56 \\
& Feuilles- Bois & 0,49 \\
& Feuilles-Pierres & 0,48 \\
& Pleine eau -Bois & 0,48 \\
& Pleine eau-Pierres & 0,46 \\
\hline \multirow{2}{*}{ Noé } & Pierres-Bois & 0,31 \\
& Feuilles-Pleine eau & 0,27 \\
& Pleine eau -Bois & 0,46 \\
& Pierres-Bois & 0,58 \\
& Feuilles- Bois & 0,54 \\
& Feuilles-Pierres & 0,36 \\
\hline
\end{tabular}

L'analyse du peuplement algal des rivières prospectées fait ressortir la prédominance des Diatomées aussi bien dans les communautés algale de pleine eau que celles fixées (Figure 5). Elles constituent au niveau de la rivière 
Soumié $77,1 \%$ des taxons fixés sur feuilles, $91,5 \%$ des taxons sur bois et $83,76 \%$ des taxons de pleine eau. Les Cyanobactéries représentent 12,5 et $3,7 \%$ des algues fixées respectivement sur feuilles et bois et $9,8 \%$ des taxons de pleine eau. Ce groupe est suivi des Chlorophytes avec $6,3 \%$ des taxons récoltés sur feuilles, $3,7 \%$ sur bois et $6,6 \%$ des taxons de pleine eau. Concernant la rivière Eholié, les Diatomées regroupent 94,9 et $92,6 \%$ des communautés algales fixées respectivement sur feuilles et sur bois contre $71,2 \%$ en pleine eau. Elles sont suivies des Chlorophytes avec des proportions respectives de 11,9 et $2 \%$ pour les algues pélagiques et celles fixées sur feuilles. Le troisième groupe constitué des Cyanobactéries représente 10,2\% des communautés de pleine eau, 3,1\% fixées sur feuilles et $6,2 \%$ sur bois. Dans la rivière Ehania, le groupe dominant est représenté par les Diatomées qui
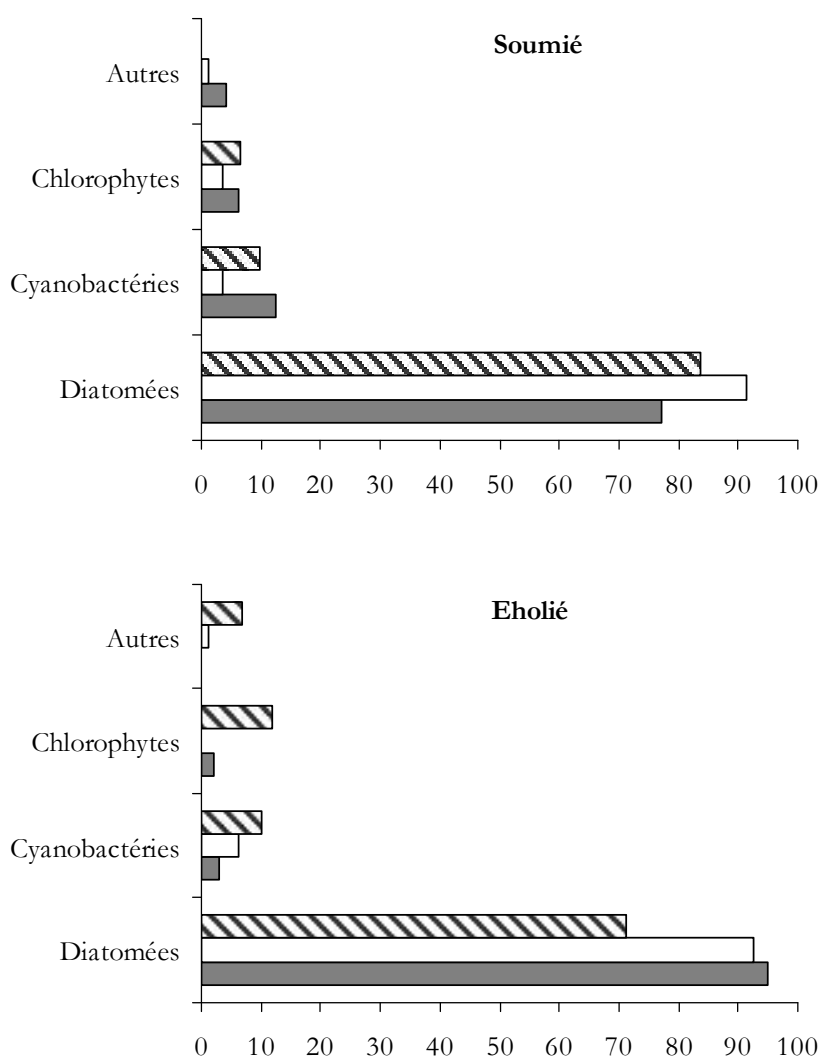

constituent $89,4 \%, 87,7 \%$ et $93,9 \%$ des taxons respectivement fixés sur feuilles, bois et pierres et $78,1 \%$ des taxons de pleine eau. Les Chlorophytes arrivent en seconde position avec $6,2 \%$ du périphyton sur feuilles, $3,5 \%$ sur bois et 6,3\% en pleine eau. Quant aux Cyanobactéries, elles constituent $9,4 \%$ des algues de pleine eau, $1,8 \%$ des algues fixées sur feuilles, $5,3 \%$ sur bois et $4,1 \%$ sur pierres. Au niveau de la rivière Noé, les Diatomées représentent $65,7 \%$ des algues de pleine eau. Elles regroupent $98,8 \%, 84,6 \%$ et $88,9 \%$ des taxons respectivement récoltés sur feuilles, bois et pierres. Les Chlorophytes représentent $3,8 \%$ des taxons fixés sur bois et $13,4 \%$ des taxons de pleine eau. Les Cyanobactéries constituent $11,9 \%$ des taxons pélagiques, $1,2 \%$ des taxons fixés sur feuilles, $9,6 \%$ sur bois et $11,1 \%$ sur pierres.
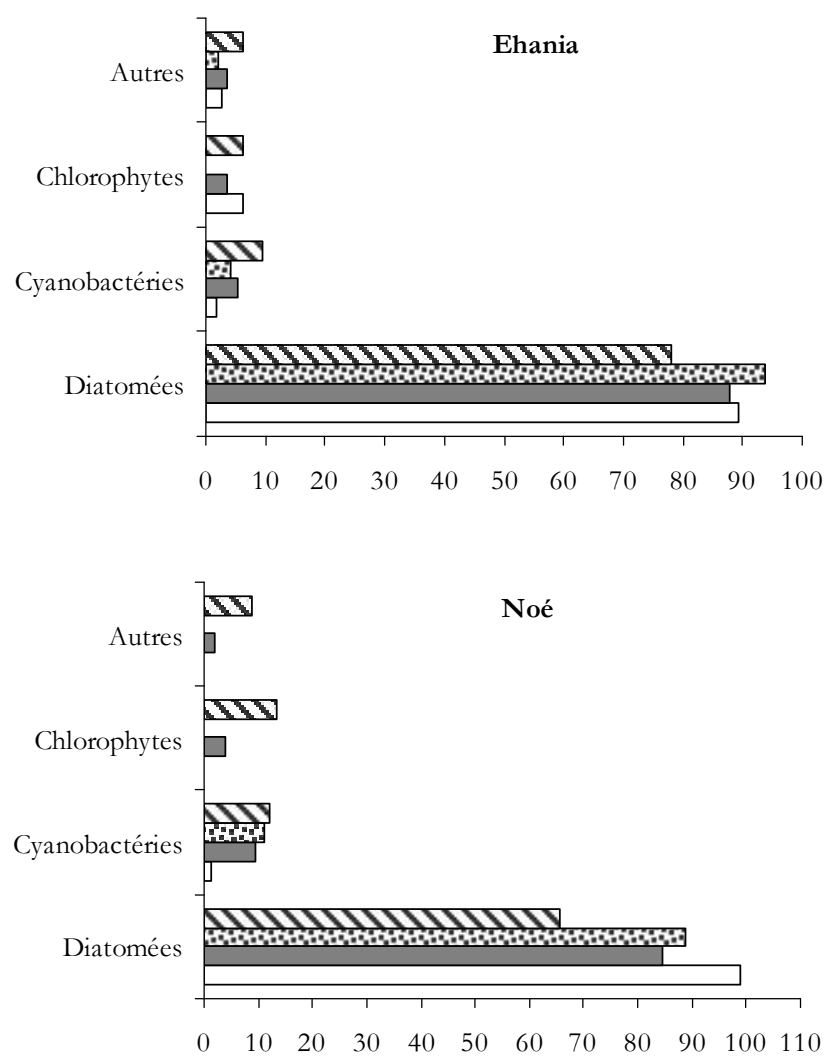

$\square$ Feuille $\square$ Bois $\quad$ P Pierre $\square$ Pleine eau

Figure 5 : Proportion des peuplements algaux périphytiques et de pleine eau des rivières Soumié, Eholié, Ehania et Noé.

Le résultat de l'analyse factorielle des correspondances basée sur la matrice présence-absence indique que les deux premiers axes factoriels expriment $47,4 \%$ de la variabilité totale (Figure 6). Ces deux axes ont été considérés pour l'ordination des échantillons. Les prélèvements de la zone pélagique sont corrélés 
positivement à l'axe 1, tandis que la partie négative de cet axe regroupe les habitats feuilles, bois et pierres (Figure 6A). Les algues recueillies sur feuilles de la rivière Soumié se démarquent des autres. Elles sont

\section{A}

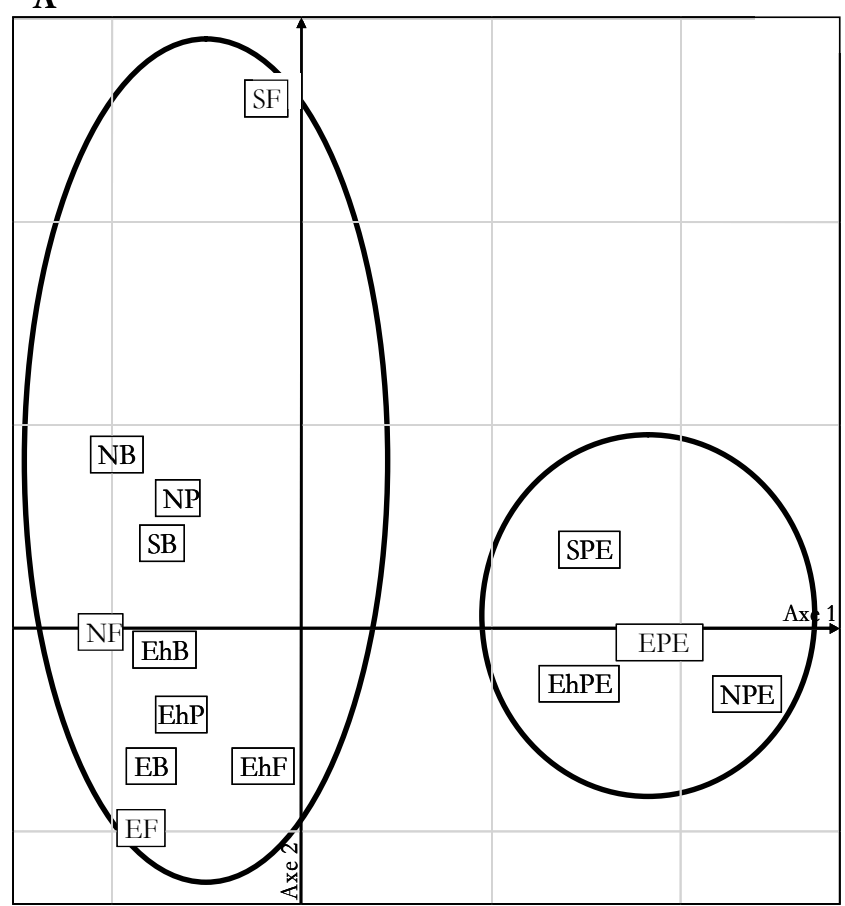

caractérisées par Eunotia faba, Anabaena sp., Lyngbya aestuarii et Luticola cohnii (Figure 6B). La partie substantielle des taxons se concentre autour de l'origine des axes.

B

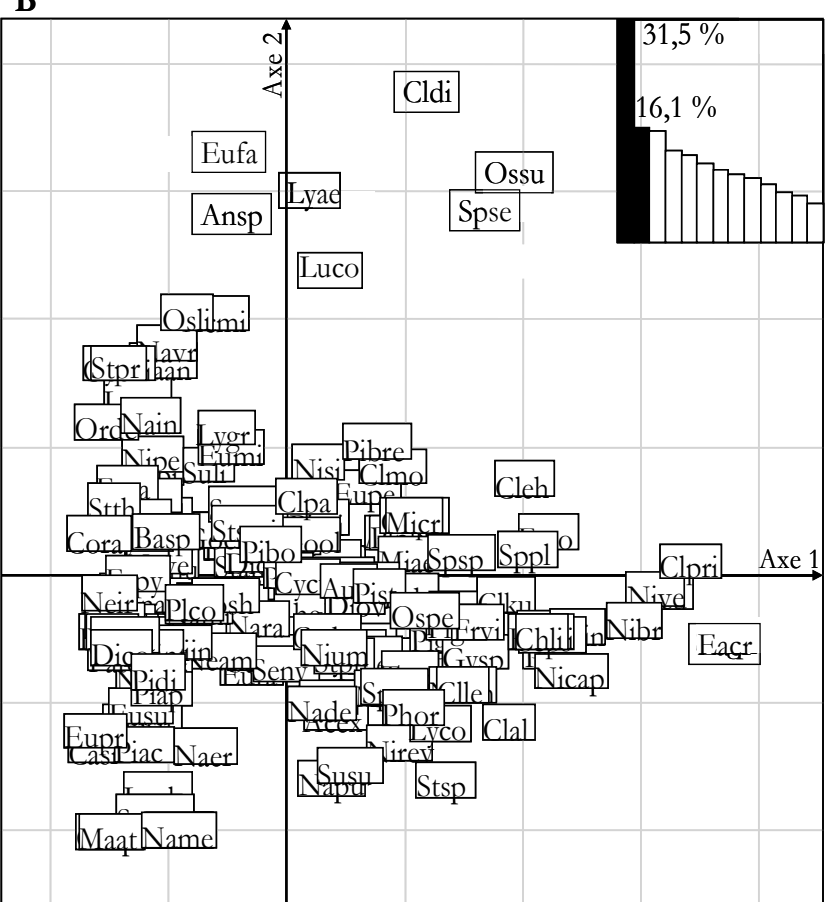

Figure 6 : Analyse factorielle des correspondances réalisée sur la matrice de présence-absence des taxons recensés $; S=$ Soumié, $E=$ Eholié, $E h=$ Ehania, $N=$ Noé, $P E=$ pleine eau, $F=$ feuille, $B=$ bois, $P=$ pierre (pour les acronymes voir tableau II).

\section{DISCUSSION}

Les valeurs des différentes variables abiotiques observées montrent que les cours d'eaux considérés ont approximativement les mêmes caractéristiques physicochimiques, malgré quelques variations observées d'une station à l'autre et d'une saison à une autre. Cette ressemblance dans les valeurs des paramètres physicochimiques peut être liée aux caractéristiques des bassins versants qui sont assez semblables dans cette région. En effet, ces rivières drainent leurs eaux sur des substrats géologiques qui ont des propriétés physico-chimiques très proches (ferrallitiques typiques et remaniés). L'absence de variations saisonnières a été également signalée par Mathuriau (2002) dans des cours d'eau du Sud-Ouest de la Colombie. Les Diatomées constituent $75,7 \%$ des taxons récoltés dans les milieux étudiés et rassemblent l'essentiel des espèces constamment rencontrées dans les échantillons. Cette dominance des Diatomées dans la composition taxonomique a été également observée par Ouattara et al. (2000) dans la rivière Agnéby et dans les zones fluviatiles de la rivière
Bia ainsi que par Round (1993) dans des cours d'eau européens. Les Diatomées sont des organismes autotrophes les plus diversifiés dans les rivières puisqu'elles ont la possibilité de coloniser toutes les surfaces disponibles. Cette facilité de colonisation justifierait leur prédominance dans la composition taxonomique des algues fixées sur feuilles, bois et pierre. En outre, les Diatomées peuvent aussi se détacher des supports et se retrouver en dérive dans la colonne d'eau, ce qui expliquerait également leur diversité importante aussi bien dans le périphyton que dans le peuplement de pleine eau. La similarité du peuplement algal entre habitats la plus élevée est observée entre les substrats constitués de feuilles et de bois dans tous les cours d'eau étudiés. Cette forte similarité serait liée au fait que ces deux types d'habitat sont des supports organiques et pourraient de ce fait être colonisés par des communautés d'algues assez proches. Par ailleurs, le peuplement périphytique des rivières côtières étudiées est dans l'ensemble plus diversifié que celui de pleine eau. 
Pourtant les algues périphytiques se retrouvent souvent en dérive après arrachage par le courant. Ce phénomène est très fréquent dans les eaux courantes (Cazaubon, 1990 ; Coste, 1996 ; Lavoie et al., 2003). Selon ces auteurs, plus le courant est élevé, plus les algues sont détachées des substrats, ce qui réduit la diversité des algues fixées. Dans les cours d'eaux étudiés, le débit $(0,1$ à $0,8 \mathrm{~m}^{3} / \mathrm{s}$ ) étant relativement faible dans l'ensemble, le phénomène d'arrachage n'est pas important, ce qui expliquerait la richesse spécifique élevée des algues périphytiques aux différentes stations échantillonnées. Cazaubon (1990) a également indiqué que les microalgues périphytiques sont plus diversifiées que le peuplement de pleine eau dans les cours d'eau européens.

En revanche, nos résultats diffèrent de ceux de Ouattara et al. (2000) sur la composition du peuplement pélagique, où ils ont recensé beaucoup plus de taxons au niveau de la rivière Bia. En effet, l'inventaire de ces auteurs prend en compte la partie lacustre de ce cours d'eau. Or le développement des algues pélagiques dépend étroitement de la stabilité de la colonne d'eau. Cette stabilité, généralement observée dans les hydrosystèmes lentiques, favorise les processus biologiques tels que les cycles complets de reproduction et de développement des algues (Ouattara, 2000). Ceci justifierait la diversité des algues pélagiques plus élevée dans les milieux lentiques comme observée par Ouattara et al. (2000) comparativement aux milieux lotiques étudiés. Les résultats de l'analyse factorielle des correspondances indiquent une différence entre les communautés d'algues pélagiques et périphytiques. Cependant, elles possèdent

\section{CONCLUSION}

La présente étude a permis de connaître la composition, la structure et la dynamique spatio-saisonnière des algues pélagiques et périphytiques en relation avec les caractéristiques abiotiques des rivières côtières Soumié, Eholié, Ehania et Noé du Sud-est de la Côte d'Ivoire. Les variations des paramètres abiotiques sont plus marquées au niveau spatial que saisonnier. A l'exception de la température et $\mathrm{du} \mathrm{pH}$, tous les paramètres mesurés varient significativement d'une station à l'autre dans une même rivière. En outre, la nature du substrat, la canopée et le mode d'occupation des bassins versants influencent la conductivité, la concentration en nitrate et le pH. Le débit quant à lui, agit sur la transparence et l'oxygénation des eaux des rivières. L'inventaire de la microflore algale a permis de recenser 192 taxons de rang spécifique et infraspécifique: 20 taxons de Cyanobactéries, 19 quelques espèces communes, ce qui indiquerait qu'il existe bien des échanges entre les algues périphytiques et les algues pélagiques. Ces échanges sont favorisés par les paramètres environnementaux tels que le débit du cours d'eau. Divers travaux réalisés sur les Diatomées d'Europe (Round, 1991), sur les algues des cours d'eau méditerranéens (Lavoie et al., 2003) et sur les Diatomées de la rivière Eholié (Niamien-Ebrottié et al., 2008) ont abouti aux mêmes conclusions. Quatre espèces sont communes à tous les habitats explorés. Ces espèces sont toutes des Diatomées pennées (ordre des pennales). II s'agit des tychoplanctoniques Ulnaria biceps, Gomphonema parvulum, Gyrosigma acuminatum et Nitzschia palea. L'ordre des pennales est composé d'algues exclusivement adhérentes et/ou fixées (Round, 1991). La grande taille de ces quatre Diatomées et leur mode de vie en colonies rubanées (Fragilaria, synonyme: Ulnaria) ou étoilées (Nitzschia), les prédisposent à l'arrachage, ce qui expliquerait leur présence dans la zone pélagique. Selon Peterson \& Stevenson (1990), la capacité des algues à rester fixées au substrat lors d'une augmentation du débit varie en fonction de leur taille, leur morphologie et leur mode d'attachement. Les Diatomées de petites tailles résistent à l'augmentation du débit et sont donc difficilement arrachées par le courant (Coste, 1996), contrairement à celles de grande taille. Nos observations corroborent celles de Fabri \& Leclecrq (1984) et Guiry et al. (2007) sur l'écologie des Diatomées. Ces auteurs indiquent que ces quatre algues sont rencontrées aussi bien dans le plancton et que dans le périphyton.

Chlorophytes, 8 taxons d'Euglénophycées, 144 taxons de Bacillariophycées et 1 taxon de Rhodophycées. Les Bacillariophycées constituent tant dans le phytoplancton $(74,69 \%)$ que dans le périphyton $(89,94 \%)$ la composante la plus importante de la flore algale dans les rivières étudiées. Les compositions algales des quatre cours d'eau ont montré de nombreuses similitudes du fait de leur température et $\mathrm{pH}$ assez semblables.. Le nombre de taxons recensés varie de 127 (rivière Éhania) à 134 (rivière Noé). Les espèces Gyrosigma acuminatum, Gomphonema parvulum, Nitzschia palea et Ulnaria biceps ont été fréquemment rencontrées dans l'ensemble des rivières considérées. Les communautés algales des stations en amont comparées à celles obtenues dans les stations en aval des cours d'eau ont montré peu de différences tant au niveau phytoplanctonique que 
périphytique. Le peu d'espèces qui les différencie ne permet pas de mettre en évidence une variation suivant le gradient amont-aval. Les résultats de cette étude indiquent que les rivières Soumié, Eholié, Ehania et Noé sont peu polluées. Cependant, pour prévenir des risques de perturbations du fait du mode d'occupation des

\section{REFERENCES BIBLIOGRAPHIQUES}

AFNOR, 1994. Qualité de l'eau. Environnement. Association Française de Normalisation. 1ère édition AFNOR, Paris, 861pp.

Anonyme 2, 2001. Recensement National de l'Agriculture (RNA) 2001. Région de Sud-Comoé. Ministère d'État, Ministère de l'Agriculture. Direction des statistiques, de la documentation et de l'informatique, $29 \mathrm{pp}$.

Arab A, Lek S, Lounaci A and Park YS, 2004. Spatial and temporal patterns of benthic invertebrate communities in an intermittent river (North Africa). Annales de Limnologie - International Journal of Limnology, $40: 317$ - 327.

Carmichael WW, 1994. The toxins of cyanobacteria. Scientific American 270: 78-86.

Carter JR and Denny P, 1987. Freshwater algae of Sierra Leone IV. Bacillariophyceae: Part II diatoms from the coastal region of southern province. Nova Hedwigia 44(1/2) : 229 - 275.

Cazaubon A, 1990. Diatomées benthiques et en dérive d'un cours d'eau méditerranéen, deux communautés distinctes ? Ouvrage dédié à $\mathrm{H}$. Germain, Koeltz : 19 - 26.

Cocquyt C, 1998. Diatoms from the Northern Basin of Lake Tanganyika. J. Camer (Ed) Berlin. 274pp, $56 \mathrm{pl}$.

Compère P, 1975. Algues de la région du Lac Tchad. III. Rhodophycées, Euglénophycées, Cryptophycées, Dinophycées, Chrysophycées et Xanthophycées. Cahier de l'ORSTOM, Série Hydrobiologie 9(3) : 167 - 192.

Compère $P, 1977$. Algues de la région du lac Tchad. VII. Chlorophytes : 3ème partie : Desmidiées. Cahier de l'ORTOM Série Hydrobiologie 11(2) : 77 177.

Compère $P$, 1980. Algues de l'Aïr (Niger). Bulletin National Plantentuin de Belgique 50: 269 - 329.

Compère $P, 1991$. Contribution à l'étude des algues de Sénégal. 1- Algues du lac de Guiers et du BasSénégal. Bulletin du Jardin Botanique National Belgique 61(3/4): 171 - 267. bassins versants, une surveillance de la qualité des eaux est nécessaire. Au plan bioécologique, cette surveillance pourrait être mise au point à travers l'établissement d'indices biologiques basés sur les algues (Diatomées et Cyanobactéries).

Compère $P, 2000$. Clé provisoire pour la détermination des genres de diatomées d'eau douce [Version 5 - V-2000] (http : //clcli.club.fr/ADLaF_cle_des genres.htm).

Coste M, 1996. Diatomées et médecine légale: Applications de la recherche des diatomées au diagnostic de la submersion vitale. Diatoms and forensic science. Edition Lavoisier Technique et documentation, Paris. 256pp.

Da KP, 2007. Étude taxinomique du phytoplancton dulçaquicole des masses d'eau lentiques et lotiques de quelques sites au sud de la Côte d'Ivoire, entre les fleuves Bandama et Bia: apports de la microscopie électronique à balayage. Thèse d'État Université de Cocody, Abidjan, 402 pp.

Da KP, Mascarell G, Couté A, 2009. Étude au microscope électronique à balayage du genre Trachelomonas (Euglenophyta) dans le Sud-est de la Côte d'Ivoire (Afrique de l'Ouest). Cryptogamie, Algologie 30(1) : 31-90.

Da KP, Traoré D, Assémien AP, 1999. Cyanophytes, Rhodophytes et Pyrrhophytes de la mare et du complexe piscicole du Parc National du Banco (Côte d'Ivoire). Systematics and Geography of Plants 69: 161-183.

Dajoz R, 2000. Précis d'écologie. 7ème Edition. Dunod, Paris. 615pp.

Desikachary TV, 1959. Cyanophyta. Indian Council of Agriculture research, New Dehli, 689 pp.

Eldin M, 1971. Le climat. In Avenard JM, Eldin M, Girard G, Sircoulon J, Touchebeuf P, Guillaumet JL, Adjanohoun E, Perraud A (Eds.) : Le milieu naturel de Côte d'Ivoire. Mémoire ORSTOM, Paris, $50: 73-108$.

Fabri $R$ and Leclecrq L, 1984. Étude écologique des rivières du nord du massif Ardennais (Belgique) : Flore et végétation de diatomées et physico-chimie des eaux. Robertville, Stat. Scient. Hautes-Fagnes. 379 pp., 33 pl. 
Foged N, 1966. Freshwater diatoms from Ghana. Biologiske Skrifter Kongelige Danske Videnskabernes Selskab 15(1): 1 - 169.

Girard G, Sircoulon J, Touchebeuf P, 1971. Aperçu sur les régimes hydrologiques. In Avenard JM, Eldin M, Girard G, Sircoulon J, Touchebeuf P, Guillaumet JL, Adjanohoun E, Perraud A (Eds.) : Le milieu naturel de la Côte d'Ivoire. Mémoire ORSTOM, Paris, 50: 109 - 155.

Guiry M. D. \& Guiry G. M., 2007.Algaebase.World-wide eletronic publication ; National University of Ireland, Galway. http://www.algaebase.org. Date de consultation juillet 2008.Anonyme 1, 2005. Données de pluviométrie de la région d'Ayamé (barrage hydroélectrique d'Ayamé, Compagnie Ivoirienne d'Electricité). Rapport d'activité.

Guiry MD and Guiry GM, 2007. Algaebase. Worldwide eletronic publication; National University of Ireland, Galway. http://www.algaebase.org.

Hyslop EJ, 1980. Stomach contents analysis, a review of methods and their application. Journal of Fish Biology 17: 411 - 429.

Ihaka R and Gentleman R, 1996. R: a language for data analysis and graphics. Journal of Computational and Graphical Statistics 5: 299 - 314.

Iltis A, 1982. Peuplements algaux des rivières de Côte d'Ivoire. II. Variations saisonnières des biovolumes, de la composition et de la diversité spécifique. Revue d'Hydrobiologie Tropicale 15(3): 241 - 251.

Jahn R and Kusber WH (eds), 2007. Algaterra Information system (online). Botanic Garden and Botanical Museum Berlin-Dahlem, FU-Berlin. http://www.algaterra.org.

Kelly MG, 1997. Use of the Diatom Index in monitor eutrophication in rivers. Water Research 36: 236-242.

Komárek J and Anagnostidis K, 1999. Cyanoprokaryota. 1. Teil : Chroococcales in Süßwasserflora von Mitteleuropa 19/1. Gustav Fischer. 548pp.

Komárek J and Anagnostidis K, 2005. Cyanoprokaryota 2. Teil/ 2nd Part: Oscillatoriales. In : Büdel B, Krienitz L, Gärtner G, Schagerl M (Eds), Süsswasserflora von Mitteleuropa 19/2, Elsevier/Spektrum, Heidelberg. 759 pp.

Konan KF, Leprieur F, Ouattara A, Brosse S, Grenouillet $G$, Gourène G, Winterton P, Lek S, 2006. Spatio-temporal patterns of fish assemblages in coastal West African rivers: a Self-Organizing
Map approach. Aquatic Living Resources 19: 361 - 370.

Kotak BG, Zurawell RW, Prepas EE, Holmes CFB, 1996. Microcystin-LR concentration in aquatic food web compartments from lakes of varying trophic status. Canadian Journal of Fisheries and Aquatic Sciences 53: 1974-1985.

Krammer $\mathrm{K}$ and Lange-bertalot $\mathrm{H}, 1986$. Bacillariophyceae : Naviculacea. In : Ettl $\mathrm{H}$, Gerloff J, Heying H, Mollenhauer D (Eds): Süßwasserflora von Mitteleuropa.Stuttgart, Fischer. 2(1). 876pp, 206pl.

Large ARG., Wade PM, Pautou G, Amoros C, 1993. Producteurs et productions primaires. In: Amoros C and Petts GE (Eds): Hydrosystèmes fluviaux. Masson, Paris. 297 pp.

Lavoie I, Vincent WF, Pienitz R, Painchaud J, 2003. Effet du débit sur la dynamique temporelle des algues périphytiques dans une rivière influencée par les activités agricoles. Revue des Sciences de l'eau 16: 55 - 77.

Mathuriau C, 2002. Les macroinvertébrés des cours d'eau andins du Sud-est de la Colombie: écologie et bioindication. Thèse de Doctorat, Université Paul Sabatier, Toulouse. 309pp.

Niamien-Ebrottié JE, Ouattara A, Ouattara M, Gourène $G, 2008$. Composition and Structure of Diatoms Assemblages of a Tropical Coast River (Eholie, Ivory Coast). European Journal of Scientific Research 20(1): 44-55.

Ouattara $A, 2000$. Premières données systématiques et écologiques du phytoplancton du lac d'Ayamé (Côte d'Ivoire). Thèse de l'Université Catholique Leuven, Belgique. 200pp.

Ouattara A, Podoor N, Gourène G, 2007. Activité photosynthétique du phytoplancton dans le barrage hydroélectrique d'Ayamé I (Côte d'Ivoire). Agronomie Africaine 19(1): 63-70.

Ouattara A, Podoor n, Teugels gg, Gourène g, 2000. Les microalgues de deux cours d'eau (Bia et Agnébi) Côte d'Ivoire. Systematics and Geography of Plants 70: 315-372.

Ouattara A, Podoor n, Teugels gg, Gourène g, 2003. Etudes préliminaires de la distribution spatiotemporelle du phytoplancton dans un système fluvio-lacustre africain (Bassin Bia; Côte d'Ivoire). Hydroécologie Appliquée 23(1): 113 132.

Peterson GP and Stevenson RJ, 1990. Post-spate development of epilitic algal communities in 
different current environments. Canadian Journal of Botanic 68: 2092 - 2102.

Prescott GW, Croasdale HT, Vinzard WC, 1975. A synopsis of North American Desmids. Part II. Desmidiaceae: Placodermae. Section 1. $275 \mathrm{pp}$.

Prygiel $\mathrm{J}$ and Coste M, 2000. Guide méthodologique pour la mise en oeuvre de l'Indice Biologique Diatomées NF T 90-354. Agences de l'Eau. 134 p. +89 pl. + cd rom TAX'IBD français/anglais.

Prygiel J, Lévêque L, Iserentant R, 1996. Un nouvel indice diatomique pratique pour l'évaluation de la qualité des eaux en réseau de surveillance. Revue des Sciences de l'Eau 1: 97 - 113.

Round FE, 1991. Diatoms in river water-monitoring studies. Journal Applies Phycology 3: 129-145.

Round FE, 1993. A review and methods for the use of epilithic diatoms for detecting and monitoring changes in river water quality. Methods for the Examination of Waters and Associated Materials. HMSO Books, London. 65 pp.

Sophia MG, Dias ICA, Araújo AM, 2005. Chlorophyceae and Zygnematophyceae from the Turvo State Forest Park, state of Rio Grande do Sul, Brasil. Iheringia, Série Botanique, Porto Alegre 60(1): $25-47$.

Thioulouse J, Chessel D, Dolédec S, Olivier JM, 1997. ADE-4: a multivariate analysis and graphical display software. Statistic and Computing 7:7583. 